\title{
Kawasaki Disease- a Case Report
}

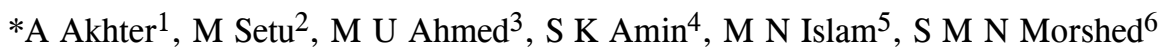 \\ ${ }^{1}$ Dr. Afroza Akhter, Assistant Professor of Pediatrics, Monno Medical College and Hospital.afroza_akhter@yahoo.com \\ ${ }^{2}$ Dr. Mumtahina Setu, Assistant Professor of Pediatrics, Anwer Khan Modern Medical College \\ ${ }^{3}$ Dr. Mesbah Uddin Ahmed, Associate Professor of Pediatrics, Gonoshasthya Somaj Vittik Medical College, Savar \\ ${ }^{4}$ Dr. Syed Khairul Amin, Professor \& Head, De partment of Pediatrics, Anwer Khan Modern Medical College \\ ${ }^{5}$ Dr. Md. Nurul Islam, Professor and Head, Department of Pediatrics, Gonoshasthya Somaj Vittik Medical College, Savar \\ ${ }^{6}$ Dr. S. M. Nahid Morshed, MD Resident, Department of Cardiology, Bangabandhu Sheik Mujib Medical University \\ *Corresponding Author
}

\begin{abstract}
Kawasaki disease is an acute necrotizing vasculitis of the medium and small-sized vessels and has life-threatening predisposition to involve coronary arteries. The diagnosis of KD still relies on the clinical criteria. We are reporting a case of classic presentation of Kawasaki disease.
\end{abstract}

Key Word: Kawasaki disease, Vasculitis

\section{Introduction}

Kawasaki disease is an acute necrotizing vasculitis of the medium and small-sized vessels, predominantly occurring in children aged 6 months-5 years, with a male-to-female ratio of $1.5-1.8$ to 1 and a lifethreatening predisposition to involve coronary arteries. As specific laboratory tests are unavailable, the diagnosis of KD still relies on the clinical criteria ${ }^{1}$. It was formerly known as mucocutaneous lymph node syndrome or infantile polyarteritis nodosa, first described by Dr. Tomisaku Kawasaki in Japan in 1967 who reported 50 cases of a distinctive illness in children seen at the Tokyo Red Cross Medical Center in Japan. Asians are at the highest risk $^{2}$. The Japanese classification for KD requires the presence of five of the following six criteria: characteristic fever, bilateral conjunctivitis, changes of lips and oral cavity, polymorphous exanthema, changes of peripheral extremities, and cervical lymphadenopathy ${ }^{3}$. Despite the availability of effective therapy to prevent cardiac complications, KD still remains an etiologic enigma. Although many epidemiologic and laboratory studies have looked at the linkage of Kawasaki disease to an infectious etiology, none of these associations has been proven ${ }^{4}$.

\section{Case presentation}

A 3 year 6 months old boy, was admitted at Gonoshasthya Somaj Vittik Medical College hospital with fever for 6 days which was high grade continued in nature, not associated with chills and rigor, rash in whole body which are diffuse and non itchy and bilateral red eye without purulent eye discharge. Parents also complained of pain in both of his knees and ankles and for which he cannot extend his knee in full range and cannot walk. He also developed swelling of his hands and feet and perianal skin excoriation. For this illness he was treated with syrup azythromycin and paracetamol for 3 days by local doctor but there was no improvement. Rinku had no significant past medical illness and received all vaccinations appropriate for his age as per EPI schedule. There was no travelling history. None of his family members were ill during or preceding his illness.

On admission, he had fever of $106^{\circ} \mathrm{F}$. His heart rate was $140 /$ minute and blood pressure was $70 / 50 \mathrm{mmHg}$. Pitting edema was present in both hands and feet. His right jogulodigastric lymph node was enlarged, $1.5 \times 1 \mathrm{~cm}$, non tender, mobile. $\mathrm{R}$ had both a blanching macular and a petechial rash on his whole body including palms and soles. He had bilateral bulbar conjunctival injection without discharge, strawberry tongue and erythematous cracked lips and his pharynx was injected without any significant tonsillar enlargement. Examination of his cardiac and respiratory systems was normal. -abdomen was soft, liver palpable, $1 \mathrm{~cm}$ below the right costal margin.

His laboratory results showed hemoglobin $9.3 \mathrm{gm} / \mathrm{dL}$, WBC 30,000/mm3, 78\% neutrophil, 20\% lymphocytes, $1 \%$ monocytes, platelet count $480,000 / \mathrm{mm} 3$, ESR 90 $\mathrm{mm} /$ hour, CRP was positive. Blood culture shows no growth, alanine aminotransferase $17 \mathrm{IU} / \mathrm{L}$, serum total protein $4.1 \mathrm{~g} / \mathrm{dL}$ and had normal ASO titer and urine analysis. His chest radiogram and ECG was normal. 
He was treated with intravenous ceftriaxone for possible bacterial sepsis and high dose $(75 \mathrm{mg} / \mathrm{kg})$ aspirin was added for Kawasaki disease. IVIG couldn't give due to financial constraint of the patient. Over the next 48 hours, he became less febrile and his rash improved. Over the next 12 hours, he became afebrile. Swelling of his hands and feet resolved in and the macular rash resolved completely within 72 hours. He was discharged after 7 days of hospitalization with low dose aspirin $(4 \mathrm{mg} / \mathrm{kg} /$ day $)$ for 8 weeks. His clinical course, hematologic parameters and was normal on follow-up. His ECG and echocardiogram were normal at 4 week after the onset of his illness.

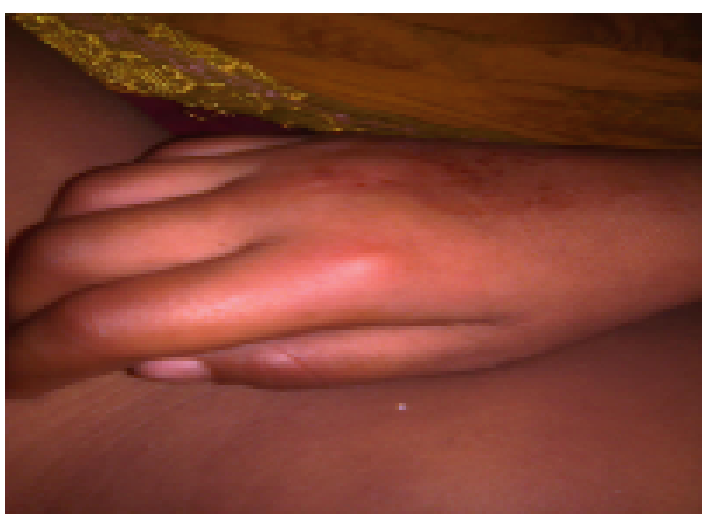

Figure-1: cracked lips with strawberry tongue

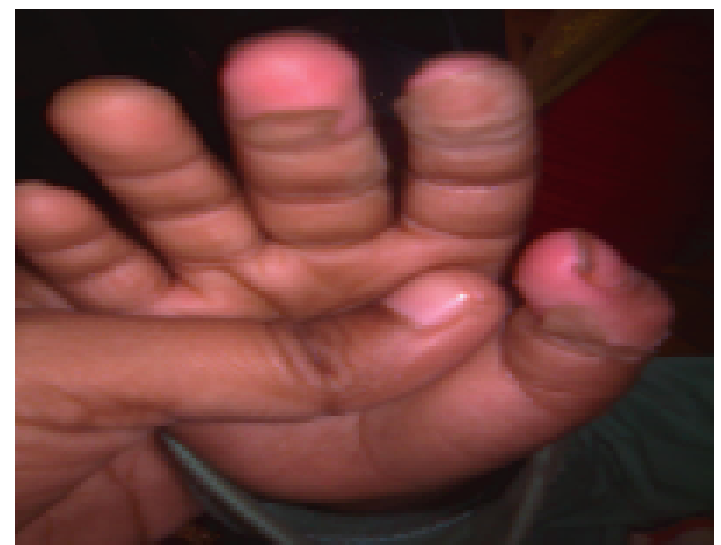

Figure-2: Edema of hands

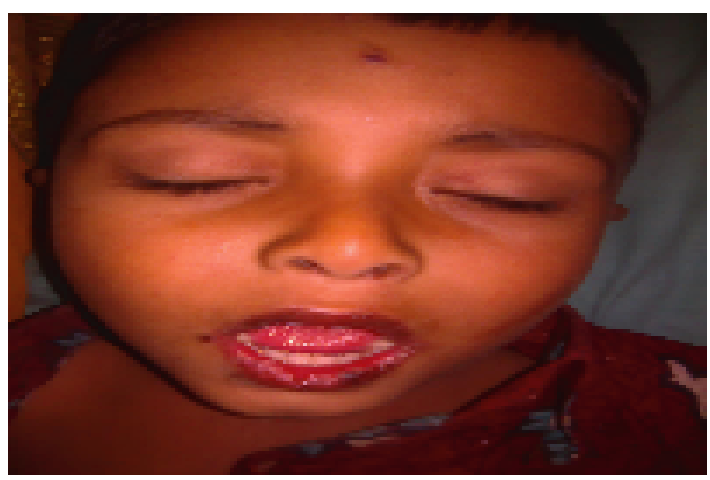

Figure-3: Bilateral conjunctival injection

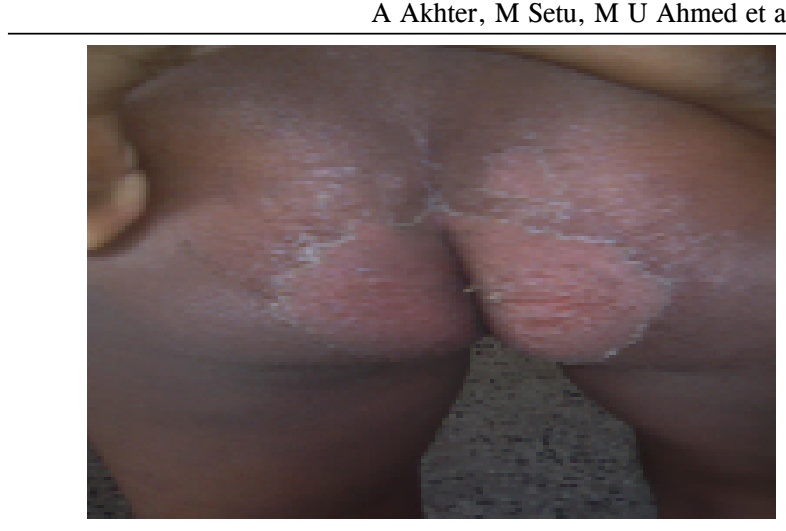

Figure-4: Perineal excoriation

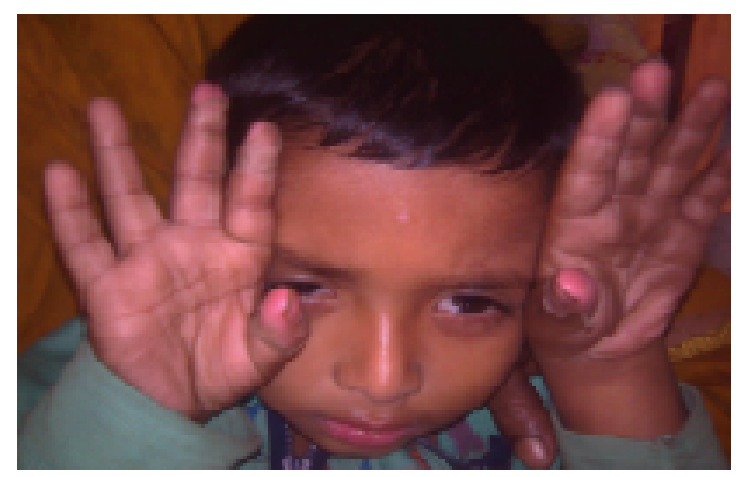

Figure-5: Periungual skin peeling

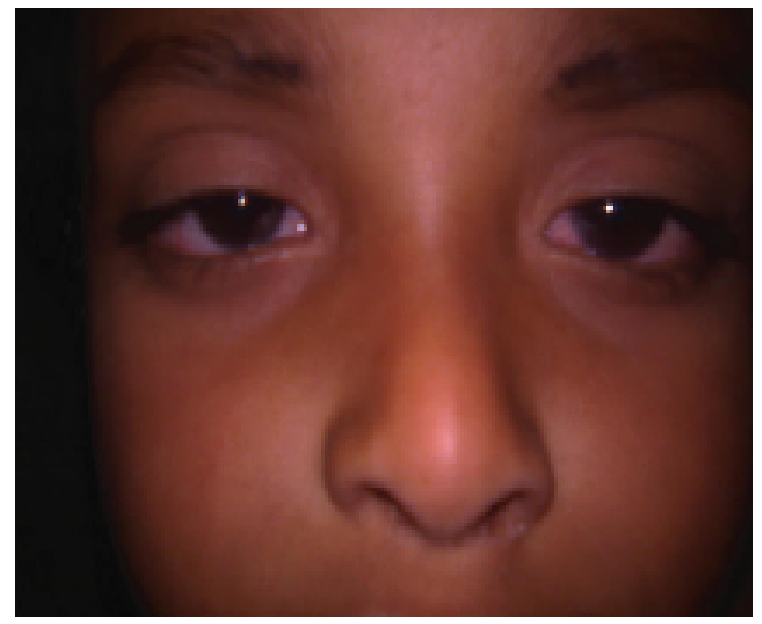

Figure -6: Periungual skin peeling

\section{Discussion}

In an English survey of family clinicians, the estimated overall annual incidence of new cases of vasculitis was 53.3 per 100,000 children under 17 years of age. The two most common vasculitides were Henoch- Schönlein purpura (HSP) and Kawasaki disease, with estimated annual incidences of 20.4 and 5.5 per 100,000 in children less than 17 years of age, respectively ${ }^{3}$. To 
Kawasaki Disease- a Case Report

date, however, an etiologic agent has not been documented. A variety of infectious agents have been proposed, including rickettsiae, viruses (primarily Epstein-Barr virus and retroviruses), Streptococcus viridans, staphylococci, Propionibacterium species and parvovirus. Standard laboratory studies have been unsuccessful in identifying a specific agent ${ }^{5}$. Recent studies have increased understanding of the etiopathogenesis of Kawasaki disease (KD). The inflammatory infiltrate in KD coronary artery aneurysms has been shown to consist of $\mathrm{CD}^{8} \mathrm{~T}$ lymphocytes, macrophages, and IgA plasma cells, consistent with an immune response to an intracellular pathogen with a mucosal portal of entry. The identification of an oligoclonal $\operatorname{IgA}$ response in the vascular wall and the detection of a KD-associated antigen in inflamed KD tissues using a synthetic antibody derived from KD oligoclonal IgA antibodies have provided new approaches to identification of the etiologic agent. Highly effective therapy has evolved for KD, even in the absence of identification of the etiologic agent. The existence of incomplete KD cases remains a significant diagnostic dilemma for the clinician6. Incomplete KD (iKD) should not be equated with mild KD. Patients with four principal symptoms were comparable to complete Kawasaki disease (cKD) with respect to CAA occurrence. In patients with one to three symptoms also, especially in those under 1 year and older than ${ }^{4}$ years of age, other significant symptoms and laboratory findings of the guidelines are very important in making a correct and early diagnosis of iKD so as to prevent coronary artery aneurysm( CAA $)^{7}$. The hidden face of the moon in this disease, the myocarditis, must not be underestimated. This myocarditis is evidenced by serial myocardial biopsy studies from patients without coronary aneurysms 8,9 .

The incidence rate and number of patients with $\mathrm{KD}$ in Japan continue to increase ${ }^{8,10}$. Epidemics of Kawasaki disease primarily occur in the late winter and spring, at 2- to 3-year intervals ${ }^{10,11}$. In our case it also occurs in late winter. The diagnosis of Kawasaki disease is clinical ${ }^{1-3,5-8}$ with a few non confirmatory supportive investigations. In our patient, we have also diagnosed him clinically with some laboratory support. In India (as also perhaps in many other developing countries), however, majority of children with KD continue to remain undiagnosed probably because of the lack of awareness amongst pediatricians. The clinical features of $\mathrm{KD}$ can be confused with other common conditions like scarlet fever and the Stevens Johnson syndrome, if the clinician is not careful ${ }^{12}$.
Treatment with IVIG relieves acute inflammation and has been shown to reduce the rate of coronary aneurysms from greater than $25 \%$ in untreated patients to $1-5 \%$ in treated patients. Maximal benefits are seen when IVIG is given within the first 10 days of the illness. Some controversy exists about the ideal time to begin IVIG, but it is given most often from days 5-7, dose is $2 \mathrm{~g} / \mathrm{kg}$ over $10-12$ hours. Most experts use highdose aspirin for a variable period, followed by lowerdose aspirin for its antiplatelet effects. High-dose aspirin $(80-100 \mathrm{mg} / \mathrm{kg} / \mathrm{d}$ orally in 4 divided doses) is given in the acute phase for its anti-inflammatory effects. It is continued until day 14 of the illness or until the patient has been afebrile for 48-72 hours. Once the patient has remained afebrile for 48-72 hours, low-dose aspirin is initiated for its antiplatelet activity. The dose is 3-5 $\mathrm{mg} / \mathrm{kg} /$ day for a total of 6-8 weeks as long as the patient shows no evidence of coronary abnormalities ${ }^{1,2,5,6.9 .13}$. We also treat our patient with high dose followed low dose aspirin. For patients who have aneurysms, aspirin should be continued until the aneurysm resolves or should be continued indefinitely. Our patient's does have any cardiac abnormality on ECG and Echo. In addition to aspirin in treatment of IVIG-resistant Kawasaki disease, corticosteroids have been proposed as part of primary therapy. Findings from the landmark Randomised controlled trial to Assess Immunoglobulin plus Steroid Efficacy (RAISE) study suggest that patients with resistance to intravenous immunoglobulin might benefit from a prolonged course of corticosteroid therapy ${ }^{13}$. Investigators are now challenged to find better techniques to identify high-risk patients in all populations with Kawasaki disease who would benefit from such a regimen.This indication are controversial, however. Schedule the patient for repeat echocardiography 21-28 days after the onset of fever. We have also done it in our patient which is normal. If baseline echocardiographic studies and those obtained at 3-4 weeks do not show any evidence of coronary aneurysms, further echocardiography is usually unnecessary, as are activity restrictions or medications beyond 3 months after the initial illness. However, a repeat echocardiogram at 1 year and a cardiovascular risk assessment at 5-year intervals are optimal. Recurrence is unusual: the recurrence rate in Japan is 3\% and approximately 1\% in North America. Most relapses occur within 2 years from the initial episode. With prompt treatment, the prognosis is good. With prompt treatment, the prognosis is good. The mortality rate among boys with Kawasaki disease in Japan is twice that among healthy boys of same age, and most deaths occur within two months of diagnosis. The mortality rate among girls with the disease appears similar to that among healthy girls, although the numbers 
are very small 14 . The average mortality rate in Japan is $0.1-0.3 \%$. Data are limited, but in the United States, death occurs in approximately $1 \%$ of affected children. In children younger than 1 year, the mortality rate may exceed $4 \%$. In patients aged 1 year or older, the death rate is probably less than $1 \%$.

\section{Conflict of interest: no conflict of interest}

\section{References}

1. F Falcini, S Capannini, D Rigante. Kawasaki syndrome: an intriguing disease with numerous unsolved dilemmas. Pediatric Rheumatology 2011; 9:17

2. Shah I. Kawasaki's Disease an Unusual Presentation. Pediatric Oncall [serial online] 2004; 1 . Art \# 12.

3. Islam M I, Talukder M K, Rahman S A. Childhood Vasculitis - An Update. Bangladesh J of Child Health 2012; 36 (1): 30-35

4. Aswine K Bal, Steven W Kairys. Kawasaki disease following Rocky Mountain spotted fever: a case report. Journal of Medical Case Reports 2009; 3:7320 .

5. Kathryn A. Kawasaki Disease. Am Fam Physician. 1999 Jun 1;59(11):3093-3102.

6. Stanford T Shulman, Anne H Rowley. Advances in Kawasaki disease. Europ J of Pediatrics 2004;163,6,285-291.
A Akhter, M Setu, M U Ahmed et al

7. Sonobe T. Prevalence of coronary artery abnormality in incomplete Kawasaki disease. Pediatr Int. 2007 ;49(4):421-6.

8. Dahdah N. Not Just Coronary Arteritis, Kawasaki Disease Is a Myocarditis, Too. JACC 2010; 55,14,1507

9. Newburger J W. Diagnosis, Treatment, and Long-Term Management of Kawasaki Disease: A Statement for Health Professionals From the Committee on Rheumatic Fever, Endocarditis, and Kawasaki Disease, Council on Cardiovascular Disease in the Young, American Heart Association Pediatrics 2004;114;1708-1733.

10. Nakamura Y. Epidemiologic Features of Kawasaki Disease in Japan:Results of the 2007-2008 Nationwide Survey. J Epidemiol 2010;20(4):302-307 .

11. Elena Corinaldesi, MFabi, G Savorelli. Kawasaki Disease in a Northern Region of Italy. Pediatr Rheumatol 2011; 9(Suppl 1):P81.

12. Singh S, Kawasaki T. Kawasaki Disease - An Indian Perspective. Indian Pediatrics2009; 46,563-571.

13. Son M B F, Newburger J W. Management of Kawasaki disease: Corticosteroids Revisited. The lancet.2012;379,1571-1572.

14. Nakamura Y et al. Mortality Among Children with Kawasaki Disease in Japan. The New Eng J med 1992;326,19,12461249. 